\title{
A prospective study of urinary tract infections in children
}

\author{
Sanath P Lamabadusuriya ${ }^{1}$ \\ Sri Lanka Journal of Child Health, 2001; 30: 31-6
}

(Key words: urinary tract infections, vesico-ureteric reflux, children, renal damage)

\begin{abstract}
Objective To study the pattern of urinary tract infection (UTI) in children paying special attention to initial confirmation of diagnosis by urine culture.
\end{abstract}

Design A prospective study over a period of twelve months.

Setting Private sector hospitals in Colombo.

Subjects Children presenting with a suspected diagnosis of UTI.

Investigations UTI was confirmed by a positive urine culture with a colony count of $>10^{5}$ organisms $/ \mathrm{ml}$ in a clean catch sample. Subsequent investigations included renal ultrasound scans, MCUGs and renal function tests; IVUs, DMSA \& DTPA scans were done in a few patients.

Results 165 patients (97 boys and 68 girls) were studied. $54 \%$ of patients were under 1 year of age. Common presenting symptoms were fever (41\%), dysuria (32\%), frequency of micturition (15\%) and failure to thrive $(8 \%)$. The commonest organisms isolated were E.coli (41\%), coliforms (38\%) and Klebsiella (13\%). The 2 most common antibiotics used initially were nalidixic acid and cotrimoxazole. On investigating further, out of 143 patients who had ultrasonography, abnormalities were seen in 17 $(12 \%)$ and in 72 patients who had MCUGs performed, 17 (24\%) showed evidence of vesicoureteric reflux (VUR). 17 patients were referred for surgical intervention.

Conclusion A diagnosis of UTI in children should be established with the "gold standard" of a significantly positive urine culture before embarking on treatment and further investigations. Special emphasis should be paid to the collection and transport of urine. Once the diagnosis is established, regular follow-up is essential to prevent renal damage.

\footnotetext{
Senior Professor of Paediatrics, University of Colombo.
}

\section{Introduction}

Urinary tract infection (UTI) is often under diagnosed and mismanaged in children, especially in developing countries like Sri Lanka with inadequate laboratory facilities. The crucial test for confirming the diagnosis is a urine culture and colony count and very few places outside the big cities in Sri Lanka have such facilities.

The incidence of neonatal bacteriuria has been reported as $1.0 \%$ to $1.4 \%{ }^{1}$. Overall, $3.7 \%$ of boys and $2.0 \%$ of girls have bacteriuria during the first year of life $^{2}$. There is male preponderance of UTI during the first few months of life. During preschool and school age females tend to predominate. The aggregate risk for symptomatic UTI up to 11 years of age has been estimated to be $3.0 \%$ for girls and $1.1 \%$ for boys ${ }^{3}$.

Approximately half of girls and two thirds of boys experience high fever with these infections. In fact, UTIs have recently been described as one of the most common serious bacterial illnesses among febrile infants and young children with a reported prevalence ranging from $4.1 \%$ to $7.5 \%$. These figures suggest that the true frequency of UTIs in children have been underestimated in the past ${ }^{2}$. It is more common in the uncircumcised male infant.

Most infections are due to colonic bacteria and are due to invasion up the urethra. Of these E.coli is by far the most commonly isolated organism, being responsible for approximately $80 \%$ of UTIs ${ }^{2}$. E.coli have recognised virulence factors which aid in the persistence of bacteria in the urinary tract and induce inflammation. Such factors include the presence of pili or fimbriae, $\mathrm{K}$ antigen in bacterial capsule, haemolysin and colicin production and the ability to acquire iron etc $^{2}$.

Numerous reports have documented that vesicoureteric reflux (VUR) is present in $25 \%$ to $50 \%$ (average $35 \%$ ) of children with culture-proven UTIs ${ }^{4}$; in contrast the incidence of VUR in children without UTI has been estimated to be between 0.4 to $1.8 \%{ }^{5}$. VUR, when present, continues to be the most significant single host risk factor in the aetiology of 
childhood pyelonephritis. The risk for acute pyelonephritis and subsequent renal scarring is related to the severity of VUR. Furthermore, when VUR is present, new renal scarring can be prevented successfully by keeping the patient free of infection. Despite the important role that VUR plays when it is present, several studies of children with febrile UTIs have reported that most patients (60-68\%) with abnormal DMSA renal scans demonstrating acute pyelonephritic changes do not have demonstrable VUR at the time of investigation ${ }^{6}$. Therefore it is evident that there are other important host de-fence factors and bacterial virulence which play a role in the pathogenesis of acute pyelonephritis in children.

Obstruction and other malformations of the upper urinary tract often present clinically as infections and are obvious predisposing factors to renal damage. The increased predisposition to infection results from impairment of urinary flow with resultant stasis that compromises bladder and renal defence mechanisms ${ }^{2}$. These conditions also inhibit the mechanical washout or flushing effect of effective micturition or ureteral peristalsis. All of these processes result in increased susceptibility of parenchyma to infection and damage ${ }^{7}$.

As some patients with UTI have vesico-ureteric reflux (VUR) which may be associated with intrarenal reflux (IRR), neglected UTI's could result in scarring of the kidneys due to the kidneys getting constantly sprayed with infected urine. At least 30\% of children with UTI and VUR have renal scarring. In the rapidly growing kidney (i.e under 05 years of age) neglected UTIs lead to renal scarring and chronic renal failure (CRF). Such patients may present for the first time in the 3rd or 4th decade of life with CRF. An important question is the role played by neglected or undiagnosed childhood UTI, in the pathogenesis of such disease.

\section{Objectives}

The main objective of this prospective study was to find the pattern of urinary tract infections (UTI) in children, paying special attention to the initial establishment of an accurate diagnosis, utilising the optimal facilities available in the private sector. It has to be emphasised that the method of collection of urine and transport time play a vital role in this process. On the other hand, if UTIs are diagnosed based on spurious data, patients could be subjected to unnecessary expensive and invasive investigations and given treatment until such investigations are completed. Therefore the criteria for establishing the initial diagnosis play a pivotal role in the management of this important childhood illness.

\section{Patients and method}

Patients were recruited from those seeking treatment in the private sector over a 12 month period. Majority of patients were self referrals while others were referred by general practitioners. They presented with a variety of complaints and if a urinary tract infection was suspected, a urine culture and colony count was performed combined with a full report of urine to establish the diagnosis. A diagnosis of a UTI was made when the colony count was over $10^{5}$ organism $/ \mathrm{ml}$. A patient with UTI may not have leucocytes in the urine; leucocytes may be found in urine in the absence of UTI e.g. acute glomerulonephritis. The amount of leucocytes found in urine would be determined by the speed and time of centrifugation and the depth from which the deposit is taken. In an uncentrifuged sample of urine the detection of $>10$ leucocytes per $\mathrm{mm}^{3}$ is thought to be significant ${ }^{8}$. The following precautions were taken to ensure the accuracy of such reports. Very specific instructions were given to the parents personally on a "one to one basis" regarding the collection of a midstream sample of urine in the premises of the hospital laboratory so as to minimise the risk of contamination and reduce transit time. The collection of urine was done by one of the parents. The genitalia were washed with soap and water prior to collection. In all boys and older girls a mid-stream sample was collected; in younger girls a sterile urine bag was used if a mid stream collection was not possible. Instructions were given to transfer the urine from the bag to the culture bottle soon after collection. All cultures were performed in one of three reputed laboratories in the private sector in Colombo supervised by a consultant microbiologist. If the growth revealed more than one type of organism the culture was repeated. Colony counts of $<10^{4}$ organisms $/ \mathrm{ml}$ were disregarded; counts between $10^{4}$ to $10^{5}$ organisms $/ \mathrm{ml}$ were repeated. All urine cultures at the time of initial diagnosis were collected after stopping antibiotics for at least 48 hours. All the ultrasound scans of the abdomen and micturating cysto urethrograms (MCUGs) were performed by a single paediatric radiologist to minimise observer variation and ensure consistency at evaluation. Majority of patients (91\%) were treated as outpatients. Fifteen babies presenting with high fever etc. were admitted for intravenous antibiotic therapy. Where the index of suspicion was high or when the patient was seriously ill, antibiotic therapy was started after collection of a sample of urine for culture. If the culture result was not significant, such 
patients were excluded from this study. Once the diagnosis was established, a 7 to 10 day course of antibiotics was given. A repeat urine culture was performed 48 hours after omitting antibiotics. If there was persistence of infection, another antibiotic was given based on the sensitivity pattern. Once infection was controlled a prophylactic dose of antibiotics (either nalidixic acid nitrofurantoin or cotrimoxazole) was given at night for one month and the urine culture repeated. During this period or during the acute illness an ultra sound examination of the abdomen was performed to detect abnormalities of the genitourinary tract. Once the infection had been controlled for over a month a MCUG was performed in children under 5 years. As this involved catheterisation, a 3 day multiple dose course of antibiotics was given to cover the procedure. If there was no evidence of vesicoureteric reflux (VUR) antibiotics were omitted and monthly urine cultures were repeated at least for 3 months and thereafter at 3 monthly intervals. During this period after omission of antibiotics, if a recurrence was suspected, a urine culture was performed without delay. In infants (i.e. $<12$ months) prophylactic antibiotic therapy was continued irrespective of the MCUG findings until completion of one year of age.

If a breakthrough infection occurred, it was treated with an appropriate antibiotic. If there was evidence of VUR, prophylactic antibiotics were given at night together with monthly urine cultures for surveillance, until the MCUG was repeated after 12 months. If VUR persisted, antibiotic prophylaxis was continued. Two patients with persisting Grade IV VUR were referred for surgery for re-implantation of ureters. DMSA and DTPA scans were performed only in a few patients as these facilities were not available in the private sector. DTPA scans were carried out in patients with obstruction prior to surgery.

\section{Results}

There were $97(59 \%)$ boys and 68(41\%) girls giving a total of 165 patients. Their age at initial diagnosis is given in Table I. The majority of patients (54\%) were under 01 year of age; whilst $34 \%$ were between 1 to 5 years. There were more boys in the under 1 year age group and more girls in the older age group. The presenting symptoms are given in Table 2. The commonest presenting symptom was fever (68 patients) of whom 08 had febrile seizures. Dysuria and frequency of micturition were other common symptoms of presentation whilst a few patients presented with failure to thrive, vomiting etc. Labial adhesions were found in 06 girls, which were separated and treated with local applications of oestrogen (Premarin) cream.

$\begin{array}{lcc}\text { Table 1 } & \text { Ages of patients at initial diagnosis } & \\ <01 \text { month } & 13 & 7.8 \% \\ \text { 02 months up to 01 year } & 77 & 46.6 \% \\ \text { 02nd year up to 05 years } & 56 & 33.9 \% \\ >\text { 05 years } & 19 & 11.5 \%\end{array}$

$\begin{array}{lcc}\text { Table 2 } & \text { Predominant presenting complaints } & \\ \text { Fever } & 68 & 41 \% \\ \text { Dysuria } & 52 & 32 \% \\ \text { Frequency of micturition } & 24 & 15 \% \\ \text { Failure to thrive } & 13 & 08 \% \\ \text { Febrile seizures } & 08 & 05 \% \\ \text { Haematuria } & 06 & 04 \% \\ * \text { Miscellaneous } & 12 & 07 \%\end{array}$

* [Vomiting, abdominal pain, abdominal distension, diarrhoea, poor feeding]

The pathogens isolated at initial diagnosis are given in Table 3. E.coil and coliforms accounted for the vast majority of infections (79\%), while Klebsiella was isolated in $13 \%$. The antibiotic used to eradicate the initial infection is given in Table 4. Sixty two patients were treated initially with nalidixic acid to control the infection while 46 patients received cotrimoxazole. More than one antibiotic had to be used to control the infection in 23 patients.

On follow-up, 26 patients (16\%) had recurrent infections. $90 \%$ of such patients were under 01 year and majority were boys. Of these, 8 boys benefited by having circumcision performed. 


$\begin{array}{lcc}\text { Table 3 } & \text { Pathogens isolated at initial diagnosis } & \\ \text { E.coli } & 68 & 41 \% \\ \text { Coliforms } & 63 & 38 \% \\ \text { Klebsiella } & 22 & 13 \% \\ \text { Proteus } & 05 & 07 \% \\ \text { Streptococcus } & 03 & \\ \text { Staphylococcus aureus } & 02 & \\ \text { Pseudomonas } & 02 & \end{array}$

\section{Table 4}

Nalidixic Acid

Cotrimoxazole

Co-Amoxiclav

Nitrofurantoin

Amoxycillin

Cephalexin

Cefotaxime

Piv-mecillinam

A total of 143 patients had renal ultrasonography performed. Abnormalities were detected in 17 patients (12\%); Table 5 lists the abnormalities found. 72 patients, all under 5 years of age had MCUGs performed; in 17 patients $(24 \%)$ there was evidence of VUR and details are given in Table 6. In 14 of these 17 patients the renal ultrasound scan was reported as normal, and majority of these patients had Grade I or II VUR. Majority of patients with Grade I
Initial antibiotic used

$$
\begin{aligned}
& 62 \\
& 46 \\
& 18 \\
& 16 \\
& 07 \\
& 12 \\
& 02 \\
& 02
\end{aligned}
$$

or II VUR on the MCUG had normal renal ultra sound scans and VUR would not have been diagnosed otherwise. In 03 patients with hydronephrosis, an IVU revealed PUJ obstruction in one patient, vesicoureteric junction obstruction in another and a left duplex system with hydronephrosis in the third. Details of patients referred for surgical intervention are given in Table7.

\section{Table $5 \quad$ Renal ultrasonography results (143 patients)}

Normal

Abnormal

Hydronephrosis

Fullness of P-C System

Pyelonephritic changes

Scarred kidneys

Hypoplastic kidney

$\begin{array}{ll}\text { Renal ultrasonography results (143 patients) } & 126 \\ & 17 \\ 06 \\ 07 \\ 02 \\ 02 \\ 01\end{array}$

\begin{tabular}{lc}
\hline Table 6 & MCUG results (72 patients) \\
VUR & 17 \\
Bilateral & 06 \\
Unilateral & 11 \\
Grade & 101 \\
Grade II & 13 \\
Grade III & 01 \\
Grade IV & 02 \\
P.U.Valves & 02
\end{tabular}

Table 7

Pelvi-ureteric junction (PUJ) obstruction Surgical referrals

Vesico-ureteric junction (VUJ) obstruction $\quad 01$

Left duplex system with hydronephrosis 01

Reimplantation of ureters (Grade IV VUR) 02

Circumcision

08

Labial adhesions 


\section{Discussion}

In this study 165 children with culture confirmed UTI were followed up over a 12 month period with special emphasis being given to the establishment of the initial diagnosis. If the parents are adequately counselled regarding the importance of proper collection of urine and shortening of transport time compliance is achievable. The high number of cases studied over this 12 month period (average of about 14 per month) illustrates how common UTIs are in a paediatrician's practice.

In most countries the "gold standard" for establishment of the initial diagnosis is a colony count of a single organism $>105$ organism $/ \mathrm{ml}$ in a mid stream sample of urine. Other methods that are used for collection of urine are urine bags, catheter samples and suprapubic aspirations (SPA). If a urine bag is used, it should be detached as soon as urine is voided to avoid contamination with organisms found in the perineum9. Catheterisation would require the use of a sterile disposable feeding tube (gauge 8 to $10)$, is invasive and would add to the expenditure. SPA is very useful in infancy where the bladder distends into the abdomen when filling but is not acceptable to some of our parents whose children are less ill. For SPA samples, a colony count of $>40,000 / \mathrm{ml}$ is thought to be significant10. For catheter samples a colony count of $>50,000 / \mathrm{ml}$ is said to be significant8. In the USA, DMSA scans are performed in the acute stage of acute pyelonephritis in febrile ill children to detect parenchymal damage2. Such facilities are not obtainable or achievable in Sri Lanka for the initial diagnosis.

Once the initial diagnosis is established, the parents should be counselled about the importance of compliance as lack of it may result in permanent damage to the kidneys. Under such guidance usually good compliance is achieved as illustrated in this study. During the period of this study, the majority of organisms cultured were sensitive to nalidixic acid, cotrimoxazole and nitrofurantoin. As the antibiotic sensitivity pattern tends to vary with time and place depending on the antibiotics used by medical professionals, it is advisable to be guided by the Antibiotic Sensitivity Test (ABST) pattern. Young infants and children tolerate liquid preparations of antibiotics better than tablets or capsules. In this study majority of patients were treated with nalidixic acid or cotrimoxozole both of which were available in liquid form.

There is irrevocable evidence that acute pyelonephritis can scar the kidney within a few weeks even if appropriate treatment is given 11 . The parenchymal damage could be reduced by prompt aggressive therapy. Therefore infants with UTI who are febrile and acutely ill should be admitted to hospital and aggressively treated with antibiotics such as 3rd generation cephalosporins (eg.cefotaxime).

Ultrasonography of the renal tract should preferably be performed by an expert as less obvious but significant abnormalities such as cortical scars may otherwise be missed. In centres where DMSA scanning facilities are readily available, renal cortical scars would be detected; in our practice such facilities are not readily available for patients. As the powers of resolution of the ultra sound machine would improve the quality of reporting, guidance could be obtained from the ultrasonographer regarding the place for referral. For the diagnosis of VUR, both isotope cystography or MCUG with radiological screening are used in specialised centres abroad12. In Sri Lanka only the latter facility is available. Although it gives very valuable information regarding VUR, the disadvantages are that it is an invasive procedure involving irradiation of the genitalia. An experienced paediatric radiologist would however minimise the exposure by reducing the number of films taken and combining it with screening. Repeat MCUGs should preferably be performed annually or 2 or 3 yearly depending on the degree of VUR because of the risks of irradiation and lead shields should cover the genitalia, whenever possible.

Historically the role played by the IVU in evaluating children with UTI has diminished over the years with the advent of ultrasonography and isotope scanning such as DMSA, DTPA and MAG-3. DTPA and MAG-3 scanning is used to differentiate nonobstructive dilatation of the upper urinary tract from obstructive dilatation and also in evaluating VUR, respectively. In this study IVUs were requested when hydronephrosis was suspected to be secondary to PUJ or VUJ obstruction. It is preferable to minimise the use of IVUs to lessen the risks of irradiation.

Some parents are obsessed by the size of the prepucial orifice and seek advice regarding surgical intervention. During voiding of urine even if there is ballooning of the prepuce with urine collecting beneath it; if the stream of urine is satisfactory, surgical intervention is usually not indicated; the parents should be encouraged to clean the orifice with soap and water regularly after slight retraction of the prepuce. However in this study there were 08 boys under 01 year of age who were referred for 
circumcision because it was thought that the persistence of a positive urine culture was related to a tight phimosis and contamination from organisms under the prepuce. In all these boys, the urine culture cleared up after circumcision.

Labial adhesions are known to predispose to UTIs in girls because of colonisation with faecal organisms. Therefore, in girls labial adhesions should be looked for and dealt with appropriately.

There is a definite correlation between constipation and recurrent UTIs in children14. Although a hard mass of stools may mechanically compress the bladder neck, there is frequent co-existence with dysfunctional voiding. Improvement in bowel habits generally results in a decrease in the incidence of recurrent UTIs in some patients.

\section{Conclusions}

A child with a suspected UTI should have a urine culture and colony count performed for confirmation of diagnosis and the infection controlled with an appropriate antibiotic without delay. The vital role played by the method of collection of urine and transport time has to be stressed. In acutely ill, febrile patients especially under 01 year of age appropriate intravenous antibiotics (eg. gentamicin, cefotaxime) should be started pending results to minimise potential renal parenchymal damage. In the majority of patients the organisms were sensitive to nalidixic acid, nitro-furantoin and cotrimoxaozole; nalidixic acid and cotrimoxazole were used more often because of their availability in liquid form, better tolerance and low cost. Not a single patient developed benign intracranial hypertension due to nalidixic acid therapy.

It is preferable for all of such children under 05 years of age to undergo ultrasonographic evaluation by an experienced person, to detect anatomical abnormalities and more severe forms of VUR. It is a safe, non-invasive relatively inexpensive procedure. Irrespective of the results of ultrasonography, MCUGs should be performed in them to detect Grades I and II VUR. In children over 05 year of age with recurrent UTI, these 2 investigations should be performed. Intravenous urography (IVU) is rarely indicated except in cases of suspected obstructive hydronephrosis.

In uncircumcised boys especially under 01 year of age, circumcision is beneficial to eradicate infection when the urine cultures are repeatedly positive. In girls with UTI, labial adhesions should be looked for routinely and attended to, so as to prevent persistence and recurrence of infection.

Other supportive measures such as prevention and treatment of constipation and provision of sufficient fluids to ensure an adequate urine flow, should also be implemented. In toilet-trained children, instructions should be given to void urine at regular intervals; if there is evidence of VUR, double or triple voiding of urine may be beneficial. It is a dilemma to advise how to manage children with UTI in places where basic facilities for urine culture are lacking. Some towns in Sri Lanka have collection centres for urine cultures to be sent to more reputable laboratories in Colombo. As quality assurance during transport is question-able, validity of such reports when positive are in doubt, although negative reports may be acceptable. If a delay between micturition and culture is unavoidable metabolism of contaminants can be inhibited by either refrigeration at $0-4^{\circ} \mathrm{C}$ or the addition of sodium borate to the specimen9 .It has been emphasised that young children with neglected UTIs may end up with CRF in later life requiring haemodialysis or renal transplantation. As these procedures are enormously expensive and valuable lives are lost due to lack of access to such facilities, it would be a worthwhile investment to improve the microbiological laboratory facilities in the less developed districts of Sri Lanka. Until such time, if facilities for proper investigations are not available, children with UTI should be referred to such centres to be properly investigated and treated so as to reduce the risk of developing CRF later.

\section{Acknowledgements}

The author wishes to thank Dr. I N A Gunaratne, Consultant Radiologist for performing the radiological investigations, Dr. Ranjan Dias, Consultant Paediatric Surgeon for the interventions and Mrs.Hemamali Pathmathilake for secretarial assistance.

\section{References}

1. Abbott G D. Neonatal bacteriuria: A prospective study in 1460 infants. British Medical Journal 1972; 1: 267-9.

2. Rushton $\mathrm{H}$ G. Urinary Tract Infections in Children. Pediatric Clinics of North America 1997; 44: 1133-69. 
3. Winberg J, Andersen H J, Bergstrom T, et al; Epidemiology of symptomatic urinary tract infection in childhood. Acta Paediatrica Scandinavica, 1974; 252 (Suppl.) 1: 1-20.

4. Smellie J M, Edwards D, Hunter N, Normand I C S, Prescod N. Vesico ureteric reflux and renal scarring. Kidney International 1975; (Suppl.4) : 65-72.

5. Bailey R R. Vesico ureteral reflux in healthy infants and children. In Hodson J, Kincaid-Smith P (editors.) Reflux Nephropathy, New York Masson, 1979.

6. Rushton H G, The evaluation of acute pyelonephritis and renal scarring with technetium 99m DMS scintigraphy. Paediatric Nephrology 1997; 11: 108-20.

7. Arneil G C. Urinary tract infection in children. British Medical Journal 1985; 290: 1925-6.

8. Hoberman A,Wald E.R.,Reynolds E.A.,et al; Is urine culture necessary to rule out urinary tract infection in young febrile children? Paediatric Infectious Diseases Journal 1996; 15: 304 -9.
9. White R H R. Management of urinary tract infection. Archives of Disease in Childhood 1987; 62: 421-7.

10. Ginsburg $\mathrm{C} \mathrm{M}$, McCracken $\mathrm{G} \mathrm{H}$. Urinary tract infections in young infants. Paediatrics 1982; 69: 409-12.

11. Rushton H G. Genitourinary infections: non specific infections. In Kelalis P.P., King L.P., Belman, A.B (Editors.). Clinical Paediatric Urology , 3rd ed., Philadelphia W.B.Saunders 1992.

12. Gelfand M J, Strife J, Hertzberg V S. Low grade vesico ureteral reflux. Variability in grade in sequential radiographic and nuclear cystograms. Clinical Nuclear Medicine 1991; 16: 243-6.

13. Kass E J, Majd M, Belman AB. Comparison of the diuretic renogram and the pressure perfusion study in Children. Journal of Urology 1985; 134: 92-6.

14. Neumann P Z, De Domenico IJ, Nogardy M B. Constipation and urinary tract infection. Pediatrics 1973; 52: 241-5 
